\title{
Un modèle de leucémie murine inductible
}

Malgré des progrès majeurs accomplis dans la compréhension des voies de signalisation induites par la protéine BCR-ABL, produit du gène de fusion résultant de la translocation $\mathrm{t}(9 ; 22)$ ou chromosome Philadelphie, Ph1) [1], le comportement biologique du clone $\mathrm{Ph}^{+}$chez les patients atteints de LMC (leucémie myéloïde chronique) est loin d'être compris. En particulier, on ne connaît ni l'événement déclenchant la survenue de la "crise blastique" qui survient immanquablement après plusieurs années d'évolution et conditionne le pronostic de la maladie, ni les effets induits par différentes quantités de la protéine BCR$\mathrm{ABL}$ dans les progéniteurs hématopoiétiques pluripotents. Pour répondre à ces questions, plusieurs modèles murins de la maladie humaine ont été établis, par transgénèse, ou par transduction des cellules souches murines par un rétrovirus porteur du transgène $b c r-a b l$ [2]. La transgénèse induite par $b c r-a b l$ est limitée d'une part par la mortalité embryonnaire importante qu'elle entraîne, probablement en raison de la toxicité de la protéine BCR-ABL, d'autre part parce qu'il n'a pas été possible de cibler de manière satisfaisante les cellules primitives pluripotentes. L'expression de BCR-ABL par l'intermédiaire d'un vecteur rétroviral crée certes une leucémie myéloïde très proche de la maladie humaine, mais la mortalité est importante (4-6 semaines) par surcharge tumorale. Ces modèles ne permettent donc pas d'étudier le comportement à long terme des cellules exprimant BCR-ABL, ce qui est un prérequis pour comprendre l'évolution moléculaire à l'origine de l'apparition d'une crise blastique sible pour répondre à cet impératif consiste à établir un modèle de LMC inductible, notamment en utilisant des transgènes contrôlés par des promoteurs sensibles à la tétracycline. Ce système d'induction nécessite la co-expression dans la même cellule, d'un transactivateur (TA ou tTA inverse selon le type off ou on de l'expression) $\left(m / s\right.$ 1999, $\left.n^{\circ} 5, p .755\right)$ et du gène d'intérêt, bcr-abl. Dans ce but, une équipe de Boston [3] a d'abord produit deux souches de souris transgéniques, l'une exprimant le transactivateur (tTA) sous le contrôle du promoteur MMTV et l'autre, le gène $b c r-a b l$ sous contrôle d'un promoteur CMV minimal précédé d'éléments de réponse à la tétracycline. Le croisement de ces deux souches de souris a été réalisé en présence de tétracycline dans l'eau de boisson des souris pour empêcher l'activation de $b c r-a b l$ pendant l'embryogenèse. Les auteurs ont ainsi dérivé 4 lignées de souris transgéniques «doubles", et, chez tous les animaux, l'arrêt de l'administration de tétracycline per os a entraîné une activation de l'expression de bcr-abl avec apparition d'une hyperleucocytose accompagnée d'une splénomégalie et d'une polyadénopathie, entraînant la mort environ 2876 jours après l'arrêt de l'antibiotique. Cependant, dans tous les cas, les cellules leucémiques étaient des précurseurs lymphoïdes de type pro$B$, et la lignée myéloïde n'était pas atteinte, ce qui peut s'expliquer par l'utilisation du promoteur MMTV chez les souris transgéniques tTA. Il était cependant clair que les leucémies pro-B pouvaient régresser au moins 3 fois de suite sur une période d'un an, après l'administration de tétracycline, ce qui démontre de manière formelle que, malgré la mul- tiplicité des voies de signalisation secondaires activées, la protéine BCR-ABL représente une cible thérapeutique majeure dans les leucémies exprimant bcr-abl. A noter enfin qu'une lignée de souris a présenté une leucémie mortelle malgré l'inhibition de $b c r-a b l$ sous l'effet de la tétracycline, ce qui est probablement dû à des anomalies génétiques secondaires car les cellules leucémiques n'exprimaient plus l'ARN bcr-abl. Le modèle de leucémie réversible réalisé dans cette étude n'est certes pas celui d'une phase chronique de LMC (car le ciblage n'a pu être réalisé au niveau de la lignée myéloïde) mais il est le premier démontrant l'inversion du phénotype leucémique in vivo par inhibition de BCR-ABL et ouvre des perspectives nouvelles pour des études moléculaires, et pour des approches thérapeutiques.

1. Faderl S, Talpar M, Estrov Z, O'Brien S, Kurzrock R, Kantarjian HM. The biology of chronic myeloid leukemia. N Engl J Med 1999; 341 : 16472 .

2. Pear WS, Miller JP, Xu L, et al. Efficient and rapid induction of a chronic myelogenous leukemia-like myeloproliferative disease in mice receiving P210 bcr-abl transduced bone marrow. Blood 1998; 92: 3780-92.

3. Huettner CS, Zhang P, Van Etten RA, Tenen DG. Reversibility of acute B-cell leukaemia induced by BCR-ABL. Nat Genet 2000; 24: 57-60.

\section{Ali Turhan}

Inserm U. 362, Institut Gustave-Roussy, 39, rue Camille-Desmoulins, 94805 Villejuif Cedex, France. 